EPJ Web of Conferences 73, 05011 (2014)

DOI: $10.1051 /$ epjconf/20147305011

(C) Owned by the authors, published by EDP Sciences, 2014

\title{
Tests of Chiral perturbation theory with COMPASS
}

Jan M. Friedrich ${ }^{\mathrm{a}, \mathrm{b}}$ on behalf of the COMPASS collaboration

CERN PH, Geneva, Switzerland

\begin{abstract}
The COMPASS experiment at CERN accesses pion-photon reactions via the Primakoff effect., where high-energetic pions react with the quasi-real photon field surrounding the target nuclei. When a single real photon is produced, pion Compton scattering is accessed and from the measured cross-section shape, the pion polarisability is determined. The COMPASS measurement is in contradiction to the earlier dedicated measurements, and rather in agreement with the theoretical expectation from ChPT. In the same experimental data taking, reactions with neutral and charged pions in the final state are measured and analyzed in the context of chiral perturbation theory.
\end{abstract}

\section{Pion-photon reactions as test of chiral perturbation theory}

Properties of the pions $\left(\pi^{-}, \pi^{0}, \pi^{+}\right)$are of crucial interest in understanding quantum chromodynamics (QCD) and its low-momentum expansion, chiral perturbation theory (ChPT), where the pions are identified as the Goldstone bosons emerging from the spontaneous breaking of chiral symmetry.

Pion-pion scattering has been studied in several approaches, and successfully described within ChPT. In contrast, for pion-photon interactions even the most fundamental process, i.e. Compton scattering still is a riddle. The leading structure-dependent term in this process is the polarisability, and its first experimental determinations resulted in values significantly higher than expected from most of the theoretical approaches, which motivated the present work. In addition, other pion-photon interactions with more pions in the final state came into reach, and are also studied.

\section{Primakoff technique and the pion polarisability}

Interactions of high-energetic hadrons with the nuclear Coulomb field represent a scattering off the quasi-real photon density, referred to as Primakoff reactions. The cross-section for a reaction $\pi^{-} A \rightarrow$ $X^{-} A$ on a nucleus $A$ reads, in one-photon exchange approximation (see e.g. [1]),

$$
\frac{d \sigma}{d s d Q^{2} d \Phi}=\frac{\alpha}{\pi\left(s-m_{\pi}^{2}\right)} \cdot F_{\text {eff }}^{2}\left(Q^{2}\right) \cdot \frac{Q^{2}-Q_{\min }^{2}}{Q^{4}} \cdot \frac{d \sigma_{\pi \gamma}}{d \Phi}
$$

\footnotetext{
${ }^{\mathrm{a}}$ e-mail: Jan.Friedrich@cern.ch

${ }^{b}$ on leave of absence from Technische Universität München, Germany
}

This is an Open Access article distributed under the terms of the Creative Commons Attribution License 4.0, which permits unrestricted use, distribution, and reproduction in any medium, provided the original work is properly cited. 
where $d \sigma_{\pi \gamma} / d \Phi$ is the cross-section for the subprocess $\pi^{-} \gamma \rightarrow X^{-}$with squared total energy $s, Q^{2}$ is the momentum transfer to the nucleus, $\alpha$ the fine structure constant, $m_{\pi}$ the pion rest mass, $Q_{\min }=$ $\left(s-m_{\pi}^{2}\right) / 2 p$ the minimum momentum transfer at beam momentum $p$. Form factor and higher-order QED effects are taken into account in $F_{\text {eff }}^{2}\left(Q^{2}\right)$ [2]. For pion Compton scattering $X^{-}$is again $\pi^{-} \gamma$ and

$$
\frac{d \sigma_{\pi \gamma}}{d \Omega_{c m}}=\frac{\alpha^{2}\left(s^{2} z_{+}^{2}+m_{\pi}^{4} z_{-}^{2}\right)}{s\left(s z_{+}+m_{\pi}^{2} z_{-}\right)^{2}}-\frac{\alpha m_{\pi}^{3}\left(s-m_{\pi}^{2}\right)^{2}}{4 s^{2}\left(s z_{+}+m_{\pi}^{2} z_{-}\right)} \cdot\left(z_{-}^{2}\left(\alpha_{\pi}-\beta_{\pi}\right)+\frac{s^{2}}{m_{\pi}^{4}} z_{+}^{2}\left(\alpha_{\pi}+\beta_{\pi}\right)\right)
$$

where $z_{ \pm}=1 \pm \cos \theta_{c m}$ and $\theta_{c m}$ is the scattering angle in the $\pi^{-} \gamma$ center-of-momentum system, and the pion structure enters through the electric and magnetic polarisabilities $\alpha_{\pi}$ and $\beta_{\pi}$. In the following, the sum $\alpha_{\pi}+\beta_{\pi}$ is neglected, as it is expected to be small. Then, the relative effect of the polarisability $\alpha_{\pi}=-\beta_{\pi}$ on the cross-section, Eq. (1), integrated in the small-momentum transfer region $Q^{2} \leq 10^{-3} \mathrm{GeV}^{2} / c^{2}$ and depending only on the fraction of energy transfered from the incoming pion beam to the emitted photon, $x_{\gamma}=E_{\gamma} / E_{\text {beam }}$, can be simplified into

$$
R=\frac{\sigma\left(x_{\gamma}\right)}{\sigma_{\alpha_{\pi}=0}\left(x_{\gamma}\right)}=1-\frac{3}{2} \cdot \frac{m_{\pi}^{3}}{\alpha} \cdot \frac{x_{\gamma}^{2}}{1-x_{\gamma}} \alpha_{\pi} .
$$

This formula is used to determine $\alpha_{\pi}$ from the measured photon energy spectrum in the reaction $\pi^{-} Z \rightarrow \pi^{-} \gamma Z$ on a nucleus with charge $Z$, as first employed for the Serpukhov data [3].

The COMPASS experiment deploys secondary hadron and tertiary muon beams from the CERN $450 \mathrm{GeV}$ super proton synchrotron (SPS). Its multi-purpose detector concept allows for a wide range of investigations in hadron physics, with high-precision and high-rate capable tracking, particle identification and calorimetry in both stages of the magnetic spectrometer. Descriptions of the relevant setups can be found e.g. in $[4,5]$. The data presented here have been collected in a two-week beam time in 2009, with significant improvements in the calorimetry and the trigger system based on the analysis of pilot run data from 2004. It was also concluded [6] that lead is not a favorable target material, but rather a $4 \mathrm{~mm}$ thick nickel disk was chosen as nuclear target.

The $190 \mathrm{GeV}$ negative-charge secondary SPS hadron beam contains more than $97 \%$ pions, which are distinguished from kaons by Cherenkov detectors. A unique feature of this measurement is that the beam can be rapidly switched from hadron to muon beam, and the spectrometer is well equipped for muon identification. This allows for control measurements with muon beam, where $\mu^{-} \mathrm{Ni} \rightarrow \mu^{-} \gamma \mathrm{Ni}$ is completely determined by quantum electrodynamics (QED).

Exclusive reactions are selected by energy conservation in the process $\pi^{-} \mathrm{Ni} \rightarrow \pi^{-} \gamma \mathrm{Ni}$, the upperleft graph in Fig. 1 shows the peak in $\Delta E=E_{\text {beam }}-E_{\pi^{\prime}}-E_{\gamma} \approx 0$, with a width in agreement with the simulation. The pion data show a tail of non-exclusive background from diffractive processes. Their contribution is not included in the simulation, instead it is estimated from events with identified $\pi^{0}$. The result is a fraction of about $5 \%$, slightly depending on the photon energy, and is subtracted in the further analysis.

The "Primakoff peak" is seen in upper-right Fig. 1. It would occur, according to Eq. (1), around $1 \mathrm{MeV} / c$, however in the data it is smeared with the experimental resolution of about $10 \mathrm{MeV} / c$, which is an excellent value on the scale of the incoming beam momentum of $190 \mathrm{GeV} / c$.

For the determination of the polarisability, the photon energy spectrum is examined according to Eq. (3). In the case of muon beam, the shape of the distribution is in excellent agreement with the simulation (not shown here). In the case of pion beam, the experimental spectrum has been corrected for the mentioned background and is divided by the simulation, in which the bremsstrahlung crosssection for a pointlike spin-0 boson is taken. This is shown in the middle graphs of Fig. 1. By fitting the distribution according to Eq. (3), the pion polarisability is determined from the COMPASS 2009 data to be $\alpha_{\pi}=\left(1.9 \pm 0.7_{\text {stat }} \pm 0.8_{\text {sys }}\right) \cdot 10^{-4} \mathrm{fm}^{3}$. Here, systematic uncertainties from $\pi-e$ scattering, 

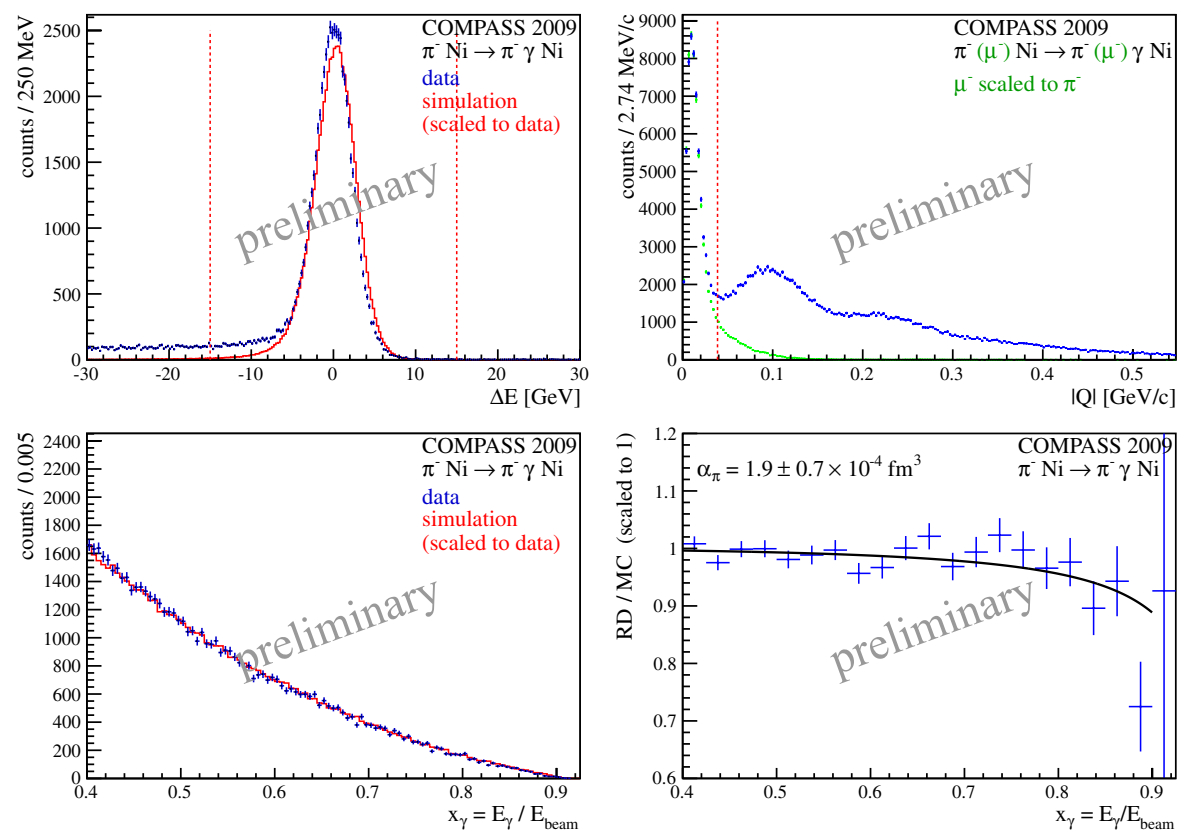

world avg.: $7.5 \pm 1.6$
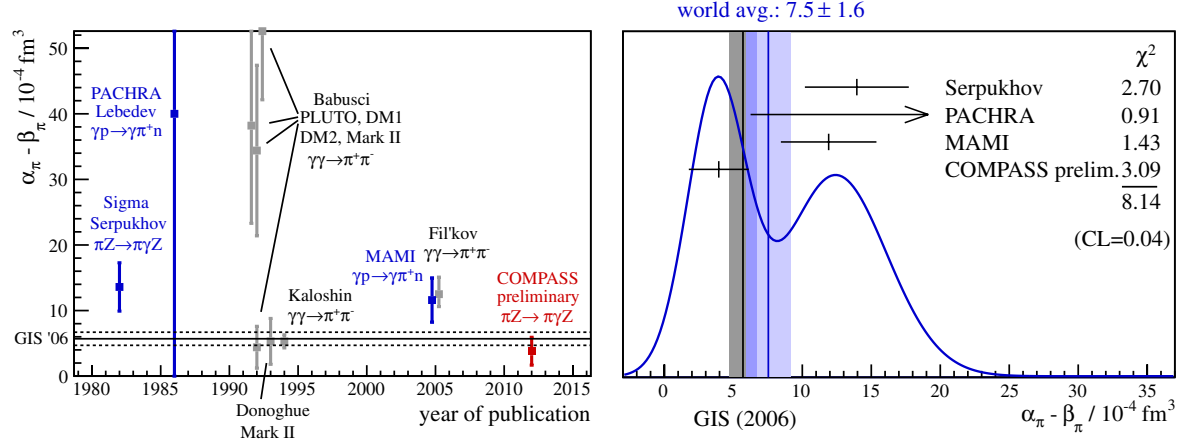

Figure 1. Upper plots: kinematic selections, as discussed in the text, are indicated by dashed lines. Middle plots: photon energy spectrum and determination of the pion polarisability. Lower plots: world data (left) and the "PDG style" ideogram [4, 17] (right). GIS(2006) is the ChPT theoretical prediction [16].

radiative corrections [6-8], apparative imperfections not accounted in the MC, and from the background subtraction have been taken into account. This value is compared to previous experimental results in the lower plots of Fig. 1. Historically (left graph) the first result [3] had been confirmed later by the dedicated experiment on radiative pion photoproduction at MAMI [9]. The available data on $\gamma \gamma \rightarrow \pi^{+} \pi^{-}$at $e^{-} e^{+}$ colliders were re-interpreted by several authors [10-13] claiming very different values for the pion polarisability, inspired by the assumptions on pion dynamics and the related low-energy constants that enter in this interpretation. Later on, it has been proven that there is no conflict between ChPT and dispersion theory $[14,15]$.

In that regard, the COMPASS result is in significant tension with the earlier experimental determinations of the pion polarisability, as the ideogram representation in Fig. 1 (right) shows, where only the dedicated experiments for the pion polarisability are included. Instead, the new result is found in good agreement with the expectation of chiral perturbation theory [16]. 
Figure 2. Mass spectrum for the reaction $\pi^{-} \mathrm{Pb} \rightarrow \pi^{-} \pi^{-} \pi^{+} \mathrm{Pb}$ in the Primakoff region $Q^{2}=t^{\prime}<0.001 \mathrm{GeV}^{2} / c^{2}$. Events from the decay of the kaons in the incident beam are seen as a peak at $m_{3 \pi}=m_{K^{-}} \approx 0.49 \mathrm{GeV}^{2} / c^{2}$. The region of interest for chiral dynamics up to $m_{3 \pi}=5 m_{\pi} \approx 0.72 \mathrm{GeV}^{2} / c^{2}$ is highlighted in grey.

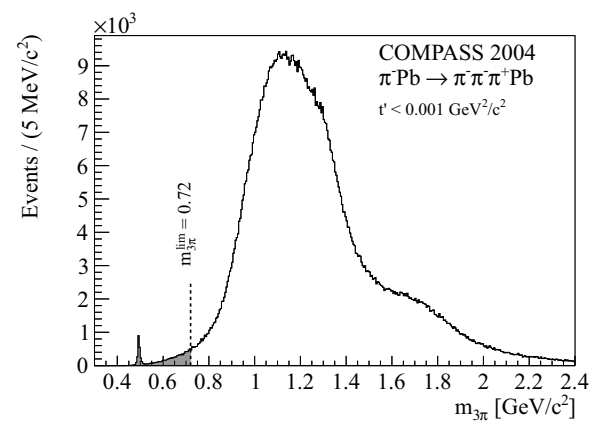

\section{Chiral dynamics in Primakoff pion production processes}

Further processes on chiral dynamics are accesible from the same experimental data [1], e.g. the chiral anomaly in the process $\pi^{-} \gamma \rightarrow \pi^{-} \pi^{0}$, however the respective analysis is still underway.

The detailed study of two-pion production at low energy is also not yet finished for the neutral-pion case $\pi^{-} \gamma \rightarrow \pi^{-} \pi^{0} \pi^{0}$. For the charged case $\pi^{-} \gamma \rightarrow \pi^{-} \pi^{-} \pi^{+}$, however, the analysis [5] of the data from the pilot run in the year 2004 has been completed. The three charged-pion final state mass spectrum obtained in Primakoff kinematics is shown in Fig. 2. The low-mass tail is of specific interest in terms of chiral dynamics, and the absolute cross-section, obtained with flux normalisation on the observed kaon decays, is found in agreement with tree-level ChPT on the level of the experimental uncertainty of $20 \%$ [18]. The neutral channel $\pi^{-} \gamma \rightarrow \pi^{-} \pi^{0} \pi^{0}$ will be of high relevance, since for this channel higherorder ChPT loop corrections are expected to play a larger role [19].

The spectrum in Fig. 2. features the salient structures from the well-known resonances $a_{1}(1260)$, $a_{2}(1320)$ and $\pi_{2}(1670)$. Their radiative couplings can be determined, which is also underway.

\section{References}

[1] N. Kaiser and J.M. Friedrich, Eur. Phys. J. A 36, 181 (2008)

[2] G. Fäldt and U. Tengblad, Phys. Rev. C 79, 014607 (2009); Phys. Rev. C 87, 029903 (2013)

[3] Yu.M. Antipov et al., Phys. Lett. B 121, 445 (1983)

[4] T. Nagel, PhD thesis, TUM(2012), CERN-THESIS-2012-138, http://cds.cern.ch/record/ 1484476

[5] S. Grabmüller, PhD TUM (2012), CERN-THESIS-2012-170, http://cds . cern .ch/record/ 1492155

[6] N. Kaiser and J.M. Friedrich, Eur. Phys. J. A 39, 71 (2009)

[7] N. Kaiser and J.M. Friedrich, Nucl. Phys. A 812, 186 (2008)

[8] N. Kaiser, Nucl. Phys. A 837, 87 (2010)

[9] J. Ahrens et al., Eur. Phys. J. A 23, 113 (2005)

[10] D. Babusci et al., Phys. Lett. B 277, 158 (1992)

[11] J.F. Donoghue and B.R. Holstein, Phys. Rev. D 48, 137 (1993)

[12] A.E. Kaloshin and V.V. Serebryakov, Z. Phys. C 64, 689 (1994)

[13] L.V. Fil'kov and V.L. Kashevarov, Phys. Rev. C 73, 035210 (2006)

[14] B. Pasquini, D. Drechsel and S. Scherer, PoS of "Chiral Dynamics 09", Bern, 037 (2009)

[15] B. Pasquini, D. Drechsel and S. Scherer, Phys. Rev. C 81, 029802 (2010)

[16] J. Gasser, M.A. Ivanov and M.E. Sainio, Nucl. Phys. B 745, 84 (2006)

[17] J. Beringer et al. [Particle Data Group], Phys. Rev. D 86, 010001 (2012)

[18] C. Adolph et al. [COMPASS Collaboration], Phys. Rev. Lett. 108, 192001 (2012)

[19] N. Kaiser, Nucl. Phys. A 848, 198 (2010) 